\title{
Análisis sistemático internacional sobre métodos reactivos, proactivos e híbridos en el tratamiento del acoso escolar
}

\author{
International systematic analysis on reactive, \\ proactive and hybrid methods in the treatment of \\ bullying
}

Teodoro Hernández de Frutos

Universidad Pública de Navarra, Pamplona, España

theo@unavarra.es

Esther Casares García

casares@unavarra.es

Universidad Pública de Navarra, Pamplona, España

\begin{abstract}
Resumen: La presión internacional, que ha llevado a reconocer el derecho de los adolescentes a desarrollar su currículo formativo en una escuela sana y segura, ha hecho que, en los últimos treinta años, los centros escolares hayan tenido que implementar planes antiacoso. El artículo presenta una revisión sistemática y actualizada de los principales métodos y técnicas antiacoso que se aplican en el mundo, estableciendo sus pros y sus contras. Básicamente, los métodos se clasifican en tres grupos: métodos integrales reactivos, para reprimir al acosador de diferentes maneras; métodos integrales proactivos, para utilizar estrategias basadas en la prevención y la solución amistosa de problemas; y métodos híbridos, no integrales o parciales, que abordan solo una parte de la intervención sin reconocer el problema en su conjunto. Se analizan los 39 métodos más importantes que se utilizan en países democráticos sobre la base de su influencia, su impronta histórica y su firmeza metodológica.
\end{abstract}

Palabras clave: Acoso escolar, victimización, intervención, métodos antiacoso, métodos integrales reactivos, métodos integrales proactivos, métodos híbridos.

Abstract: Over the last thirty years, schools have found it necessary to implement anti- bullying policies, as a result of strong international pressure to recog- 
nize the right of adolescents to receive their education in a healthy and safe environment. This article presents a systematic and updated review of the main anti-bullying methods which have been developed worldwide, highlighting their strengths and weaknesses. Broadly speaking, the methods can be categorized into those that involve comprehensive reactive strategies to repress the bullies in different ways, comprehensive proactive strategies based on the prevention and resolution of problems through mediation and negotiation, and hybrid methods, which are non- comprehensive or partial and tackle only one part of the intervention without considering the problem as a whole. The 39 most important methods developed in democratic countries on the basis of their influence, their historical significance and their methodological solidity are analyzed.

Key words: Bullying school, victimization, intervention, methods anti-bullying, reactive whole methods, comprehensive proactive methods, hybrid methods. 


\section{INTRODUCCIÓN}

El acoso escolar, en tanto que agresión y victimización, es un fenómeno mundial (Smith and Brain, 2000) expresión inequívoca de los procesos de identidad emergente que han irrumpido con fuerza en los últimos veinte años a raíz de la difusión de los efectos perniciosos que puede llegar a tener entre jóvenes, tanto individuales, provocando o favoreciendo suicidio, depresión, ansiedad, o pánico (Copeland et al., 2013), percepción del grupo de pares (Williford, 2012) o síntomas depresivos cuando son adultos (Ttofi et al., 2011); como sociales, afectando al rendimiento escolar, malas notas, desafío a la autoridad, machismo, ineficiente capital social, consumo de drogas, delincuencia, debilitamiento de vínculos sociales, desconfianza y desapego con la sociedad (Sing y Ghandour, 2012). Existen unos ocho millones de adolescentes en el mundo que padecen bullying si admitimos como buena la cifra que establece que el acoso severo lo padece alrededor del tres\% de la población adolescente. Es un hecho que se ha podido comprobar en China, Japón, Brasil, Australia, Sudáfrica, Estados Unidos, España, Angola o Colombia, por poner un ejemplo. Se repite en las escuelas como producto de una escolarización obligatoria que supone aglutinar a jóvenes en una época de desarrollo social convulsiva en la que se tienen que cristalizar identidades de género emergentes. Si el nivel de educación afecta a la productividad de un país, el bullying puede afectar a ambos ante la pérdida de recursos que supone.

El reconocimiento mundial de una evidencia que permanecía oculta y la obligación de cumplir con los compromisos internacionales de derechos de los adolescentes (ONU, 1991) han llevado a los centros escolares, unas veces obligados por ley, como en Gran Bretaña, Noruega, Finlandia, Australia y Canadá, y otras ante la presión de los padres y de la opinión pública, a implantar métodos antiacoso escolar que combinan prevención e intervención. En España, el texto consolidado de 29 de julio de 2015 de la Ley de Educación enfatiza la importancia de los métodos antiacoso al sostener, en el artículo 124 , respecto a las normas de organización, funcionamiento y convivencia, que los centros elaborarán un plan de convivencia que incorporarán a la programación general anual y que recogerá todas las actividades que se programen para fomentar un buen clima de convivencia dentro del centro escolar, así como la realización de actuaciones para la resolución pacífica de conflictos, con especial atención a las relacionadas con la prevención de la violencia de género, la igualdad y la no discriminación. Además, en el punto 2 se establece que las normas de convivencia y conducta de los centros son de obligado cumplimiento y deben concretar los deberes de los alumnos y las medidas correctoras aplicables en caso de incumplimiento, tomando en consideración su situación y sus condiciones personales. En cuanto a las competencias del director (artículo 
132), se señala que debe favorecer la convivencia en el centro, garantizar la mediación en la resolución de los conflictos e imponer las medidas disciplinarias que correspondan a los alumnos, en cumplimiento de la normativa vigente.

Los centros escolares han adquirido conciencia del problema implantando medidas de seguridad que complementan prevención e intervención basadas en métodos y técnicas anti-bullying (Cross, 2012), mientras otros autores sostienen que el trabajo con pares no es efectivo a estas edades (Ttofi y Farrington, 2011: 44). La prolífica investigación sobre bullying evidencia que es persistente a través del tiempo (Willford et al., 2012) e incluso con tendencia a aumentar (Rigby y Smith, 2011), por lo que se hace necesario intervenir.

\section{ANTECEDENTES Y FUNDAMENTACIÓN TEÓRICA}

La literatura sobre la reducción del acoso escolar por medio de programas antiacoso ha sido contradictoria e inconsistente, tanto en los resultados como en el procedimiento que había que seguir (Kljakovic y Hunt, 2016; Jiménez-Barbero et al., 2016). Sobre una base de 44 estudios, Ttofi y Farrington (2011) informaron de una reducción de entre el 20 y el $23 \%$ en acoso y entre el 17 y el $20 \%$ en victimización; los programas que incluían reuniones con los padres, disciplina y mejora de la supervisión de la zona de juegos fueron los más efectivos. Evans, Fraser y Cotter (2014), revisando 24 intervenciones sobre reducción de acoso escolar, constataron la ausencia de efectos o solo observaron efectos significativos débiles en 18 (67\%) de los casos, y notificaron que las intervenciones practicadas fuera de Estados Unidos con muestras homogéneas resultaban ser más exitosas que los programas implementados en ese país, donde las muestras tienden a ser más heterogéneas.

Yeager, Fong, Lee y Espelage (2015), después de escoger 19 estudios de una base de datos amplia, cuestionaron en un metaanálisis la efectividad de los programas antiacoso escolar a partir de los once años en lo que denominan «moderación de los efectos de los programas relativa a la evolución de la edad»: grados $1 .^{\circ}$ a $7 .^{\circ}(d=0,13)$; grados por encima del $8 .^{\circ}(d=0,01)$. Cantone et al. (2015), en una revisión sistemática de 17 trabajos de campo aleatorios, concluyeron que la mayoría de los programas no aseguraban efectos positivos y que la intervención integral era más efectiva que la dirigida hacia el currículum escolar o hacia el entrenamiento de habilidades sociales. Jiménez-Barbero et al. (2016), en una selección de 14 estudios, destacaron que la heterogeneidad de los casos complicaba mucho la evaluación, aunque llegaron a la conclusión de una cierta pero débil influencia de los programas en la reducción del acoso escolar. Lee, Kim y 
Kim (2015) también concluyeron que los efectos eran limitados después de analizar 13 estudios seleccionando la reducción del acoso.

Existen revisiones sistemáticas que no contemplan la disminución del acoso escolar, sino otras cuestiones (Kljakovic y Hunt, 2016; Van Noorden et al., 2014; Salgado, Senra y Lourenço, 2014).

No se conocen trabajos que hayan revisado sistemáticamente, sobre la base de los tres programas diferenciados que se presentan en esta investigación, aunque varios autores se han ocupado de cuestiones colaterales teniendo en cuenta características parecidas. Los conceptos de la agresión reactiva y proactiva fueron introducidos por primera vez en 1987, pero tienen unas raíces más antiguas y profundas en el campo de la investigación de la agresión. La idea surge del modelo de la frustración-agresión, que contempla esta última como una reacción hostil, airada, a la frustración percibida. Más actualmente, y en relación con el concepto de acoso escolar, Ttofi y Farrington (2011) hicieron una primera división de los programas antiacoso en disciplinarios y de pares. Smith, Salmivalli y Cowie (2012) denominaron a los programas disciplinarios reactivos, dado que algunas variables que se incluyen, como reprimendas verbales y contacto con los padres, las utilizan todos los programas y, por lo tanto, las prácticas disciplinarias no se consideran exclusivas de los programas denominados punitivos. En su contrarréplica, Ttofi y Farrington (2012) argumentaron que con programas disciplinarios se referían a incluir sanciones, pero no incorporaron el concepto de reactivos. Rigby (2013) utilizó seis categorías para agrupar todos los programas antiacoso escolar: disciplinario, tradicional, refuerzo, mediación, grupo de apoyo, práctica restauradora y decisiones compartidas.

\section{DESARROLLO TEÓRICO}

\section{Grupo 1. Programas reactivos}

Este grupo se ha elaborado a partir de los programas integrales caracterizados por intervenir en cuatro espacios diferenciados: alumnos, centro, padres e instituciones. Tiene sus raíces en el programa Olweus, experimentado en 1983, e incluye sus versiones internacionales, principalmente estadounidenses (Melton et al., 1998; Bowllan, 2011). Se puede identificar con la metodología propuesta por Olweus y su insistencia en el uso de medidas contundentes cuando se detecta un caso claro de acoso. Incorpora como principal rasgo distintivo un elemento «reactivo» o «disciplinario» categórico, porque se aplica al infractor o agresor, ya sea individual o colectivo. 
Siguiendo la propuesta de Ttofi y Farrington (2011), se impone un castigo con el objetivo de que la conducta no se repita. Se considera fundamental mantener la autoridad y mandar un mensaje inequívoco al agresor de que no se consentirán esos comportamientos; se le hace entender que el centro educativo está decidido a hablar seriamente con quien agrede, a quien podrá expulsar de la clase, enviarlo castigado a otra aula, confinarlo en la sala del profesor o tutor durante los recreos, enviarlo a hablar con la dirección, despojarlo de algunos privilegios importantes y aplicarle otras sanciones, la más grave de las cuales sería la expulsión definitiva del centro por medio de un expediente disciplinario.

Thompson y Smith (2011) denominan a este tipo de acciones «sanciones directas»; consisten en amonestación verbal al agresor, reuniones con los padres, apartamiento temporal del aula, retirada de privilegios, exclusión interna, servicio a la comunidad escolar, expulsión a corto plazo, expulsión indefinida. En un estudio basado en estas premisas y denominando a su modelo «reactivo», Gregory et al. (2010), en una muestra estatal de más de 7.300 estudiantes y 2.900 profesores, hallaron que la disciplina académica autorizada (es decir, el constante cumplimiento de normas disciplinarias justas) y la disponibilidad de los adultos se asociaban con menos intimidación y victimización, incluso después de controlar el tamaño de la clase y la proporción de minorías étnicas y estudiantes de pocos recursos. Valdés-Cuervo, Martínez-Ferrer y Carlos-Martínez (2017) mantienen que las prácticas docentes no permisivas y aquellas que implican a familias en la prevención de la violencia se relacionan con una menor agresión entre pares.

Entre las medidas destacan: actuación en el colegio, actuación en el aula, reglas contra intimidación, participación paternal, establecimiento de un protocolo para el manejo de situaciones de acoso, carteles u otros marcadores visibles de la campaña antiacoso, intensidad de aplicación adecuada (más de 20 h), materiales de plan de estudios, vídeos o actividades de ordenador, acercamiento orientado entre pares, intervención de espectadores, entrenamiento del profesor, supervisión de patio, asambleas antiacoso

\section{Grupo 2. Programas proactivos}

Dentro de este grupo se incluyen los programas que se asemejan al prototipo KIVA (Kärnä et al., 2013; Salmivalli, Kärnä y Poskiparta 2011), caracterizados por dedicar mayores recursos y por contar con la participación activa de observadores. Se han iniciado y desarrollado en Finlandia, inspirados en las iniciativas Olweus, pero en un momento dado se distinguieron por aplicar una puesta en escena más elaborada con la in- 
clusión de vídeos, internet y juegos, y por contar con personal fácilmente reconocible y dedicado exclusivamente a evitar el acoso. El resultado fue un modelo con una filosofía diferente que puede llegar a ser bastante más costosa (Clarkson et al., 2015). Son además programas integrales, porque actúan sobre cuatro aspectos: alumnos, centros, familias e instituciones. La mayor diferencia con los programas reactivos radica en que, al gestionar la resolución de conflictos, tratan de evitar las sanciones directas contra los acosadores. Su pedagogía se apoya en fomentar el mantenimiento de un ambiente de aprendizaje positivo, solidario e inclusivo. De alguna forma, estos modelos recuperan una práctica docente alternativa que surgió en las últimas décadas del siglo xx y que consistía en eliminar el castigo traumático a los alumnos y más tarde las sanciones directas. Uno de sus aspectos principales es la insistencia en que las relaciones entre alumnos y entre estos y los profesores deben basarse en el respeto mutuo. Su justificación como modelo se establece en las críticas fundamentadas hacia los programas integrales reactivos, al sostener que el uso frecuente de ese tipo de estrategias puede deberse al desconocimiento de la efectividad de la prevención o a la falta de creencia en su eficacia. Critica también la práctica escolar docente ampliamente extendida que recomienda a los centros escolares y a los profesores ser tan estrictos como sea posible al inicio del curso para luego ir rebajando poco a poco la intensidad de esa firmeza. Se sostiene que la falta de seguridad de los profesores respecto al uso de estrategias de prevención, tales como la negociación sobre las normas de aula, desemboca en técnicas reactivas severas, probablemente menos eficaces. Se utilizan las mismas medidas que los programas anteriores, pero además se confía en el uso de elementos destinados a alumnos y a profesores (cursos, charlas, libros, vídeos, trabajo colectivo), porque tanto la intensidad como la duración del programa se asocian a una disminución del acoso y de la victimización.

\section{Grupo 3. Métodos no integrales o parciales}

\section{Métodos no integrales o parciales}

Frente a los modelos anteriores, existen otros que han pretendido elaborar una manera de actuar concreta y circunscrita a un problema de violencia en el centro que, en momentos puntuales, les permitiese abordar la resolución de conflictos que abarcan desde el acoso escolar hasta otros incluso más punibles. Muchos de estos procedimientos son anteriores al reconocimiento del fenómeno del acoso escolar y se emplean tanto en los métodos integrales reactivos como en los proactivos cuando se trata de dar solu- 
ción a un problema. Su filosofía encaja más entre los métodos proactivos, porque en la medida de lo posible evitan el castigo físico o las sanciones directas. Pero son prototipos que solo persiguen la intervención, no la prevención. Algunos de ellos proceden de filosofías docentes basadas en la justicia restauradora. Todos los casos recogidos en el Cuadro 1 incluyen alguno de estos métodos e incluso la combinación de varios de ellos.

\section{El método preocupación compartida o método Pikas}

Aplicado por Anatole Pikas (1989) en Suecia, y conocido como «método de preocupación común», consiste básicamente en dos formas de abordar el acoso en función de la edad de los acosadores. En la primera, cuando se trata de casos en los que están implicados menores de nueve años, basta con que un adulto o pacificador le diga al acosador, con firmeza y autoridad, que su comportamiento debe cesar (método directo). En la segunda, en la que se encuentra implicado un grupo o una banda, lo apropiado es reindividualizar a los participantes, dado que los pensamientos y sentimientos del grupo son más simples que los de sus miembros, y encauzarlos preocupándose por la persona que ha sido agredida y cuidando de ella. Se contemplan tres fases: una entrevista individual con cada uno de los acosadores implicados (entre 5 y 10 minutos por acosador), en la cual se trata primero al principal implicado o cabecilla; una indagación consistente en preguntar a los agresores cómo está la situación y qué pueden hacer para ayudar, y en la cual si existen propuestas, se aceptan, y si no las hay, se sugieren soluciones; una entrevista al acosado, en la que se lleva a cabo una fase de seguimiento, estableciendo finalmente una reunión para evaluar cómo se ha llevado a cabo todo el proceso y reforzar el comportamiento modificado.

\section{Método de grupo de apoyo o no inculpación}

Aplicado por Hopkins, Maines y Robinson (2011), se apoya en el argumento de que la violencia surge de las relaciones interpersonales en las que el dominio y el estatus se consiguen a través o a expensas de los miembros menos prestigiosos del grupo. Se establece un grupo de apoyo formado por los acosadores y los observadores implicados en un incidente determinado, responsabilizándolos del hecho, en vez de culpabilizándolos directamente. Se entrevista a la víctima primero para descubrir qué ha ocurrido, en qué ha consistido la intimidación sufrida y quiénes han sido los responsables. A continuación, se convoca una reunión con los alumnos implicados, a los que se les comunica que por su especial situación pueden ayudar a la persona afectada. Entre los presentes en la reunión 
también se incluye a estudiantes que han sido seleccionados porque se espera que su apoyo a la víctima sea relevante. A ese grupo se le informa sobre lo que ha sucedido y sobre cómo se encuentra el estudiante, tratando de conseguir que empaticen con la víctima. Se les transfiere la responsabilidad de ayudarla. Se pide al grupo ideas y actuaciones para conseguir la plena incorporación del acosado al centro y que se sienta protegido y comprendido. La víctima nunca está presente. Se deja que el grupo ponga en práctica su plan y una semana después el profesor o interventor se reúne con cada uno de los miembros del grupo. Si el acoso ha desaparecido, se felicita al grupo y se disuelve. En definitiva, lo que se sostiene es que la violencia es un fenómeno que surge de un particular tipo de cultura grupal que persigue crear una vinculación especial entre agresores y víctimas.

\section{El método de los círculos de calidad}

Incluido en el modelo Sheffield, enfatiza especialmente los canales de comunicación en la escuela. Se forman grupos de cinco o seis miembros que realizan reuniones periódicas, normalmente semanales, y que están habilitados por alguien con experiencia en la identificación de problemas, en su análisis y en la búsqueda de soluciones, que deben presentar al equipo directivo, en este caso al del colegio. Se adiestra a los componentes de los equipos para resolver problemas mediante la generación de ideas, la observación y la recolección de datos, y la aplicación de estrategias para buscar soluciones y comunicarlas. El ciclo consta de los siguientes pasos: formar el grupo, hacer una lluvia de ideas sobre el problema, darle prioridad e investigarlo, identificar las causas, sugerir soluciones, presentar el problema y las soluciones a la Dirección del centro, monitorizar y evaluar los resultados.

\section{El método de apoyo de pares}

Este método, pionero en el Reino Unido en la década de 1990, consiste en entrenar a los estudiantes en habilidades básicas como una parte crítica de una política escolar antiacoso. Según Cowie y Hutson (2005), parte de la premisa de que existen potencialidades en la gente joven que se pueden desarrollar para ayudar a sus congéneres. Se basa en tres puntos: primero, se entrena a los jóvenes para trabajar juntos fuera de sus grupos de amigos. Este tipo de interacción ayuda a reducir prejuicios y promueve la confianza a través de género y los grupos étnicos. Segundo, a través del entrenamiento se da una oportunidad a los jóvenes para aprender habilidades comunicativas, para compartir in- 
formación y para reflexionar sobre sus propias emociones en relación con los demás. Y tercero, se entrena a los jóvenes para abordar los conflictos y para ayudar a sus compañeros a relacionarse entre sí de una manera más constructiva, no violenta.

Algunas de las características de los principales programas se pueden observar en el Cuadro 1.

\section{DISEÑO Y METODOLOGÍA}

Para documentar los estudios sobre bullying, especificando el tipo de intervención, se acudió a publicaciones referenciadas en las principales bases de datos: ASSIA, BASE, Campbell Collaboration, Cochrane Library, Dissertation Abstracts, ERIC, Google Académico, Google Books, International Data Archives, ISBN, ISOC, IBSS, Index to Thesis Database, PsycInfo, PubMed, Social Sciences Citation Index, Social Services Abstracts, Blacwell Encycopedia, Dialnet, ProQuest Sociology, Scielo, SocIndex, SSRN, Elsevier Data Search, Emerald, Encyclopedia of Adolescence, Francis, Sociological Abstracts, Social Work Abstracts, y Scopus. Se seleccionaron libros, artículos de libros y artículos en revistas. De todas las aportaciones se escogieron las más relevantes para nuestro objeto de estudio por ser las más reconocidas, las más solventes a nivel metodológico y por ser representativas de diferentes países. Se trató de dar una perspectiva internacional recogiendo al menos alguna intervención en un país, o cuando el país es de una gran dimensión, de varios de sus estados. Se analizó cada método utilizado desde el punto de vista de las características organizacionales y del éxito en la reducción de acoso y de victimización. El punto de partida fue el estudio seminal llevado a cabo por Olweus en Noruega. Un requisito para incluir los casos en la revisión sistemática internacional ha consistido en el requerimiento de que incorporen lo más nítidamente posible los efectos de los programas comparando un grupo experimental que recibió la intervención con un grupo control que no la ha recibido. Entendiendo por grupo experimental o semiexperimental a los estudiantes que se expusieron al programa independientemente de su asignación al azar.

\section{CRITERIOS DE INCLUSIÓN Y EXCLUSIÓN}

Para poder llevar a cabo una revisión sistemática de casos con las suficientes garantías se establecen las condiciones de búsqueda según los criterios AMSTAR (Assessment of Multiple SysTemAtic Reviews), instrumento utilizado en la medición en este tipo de revi- 


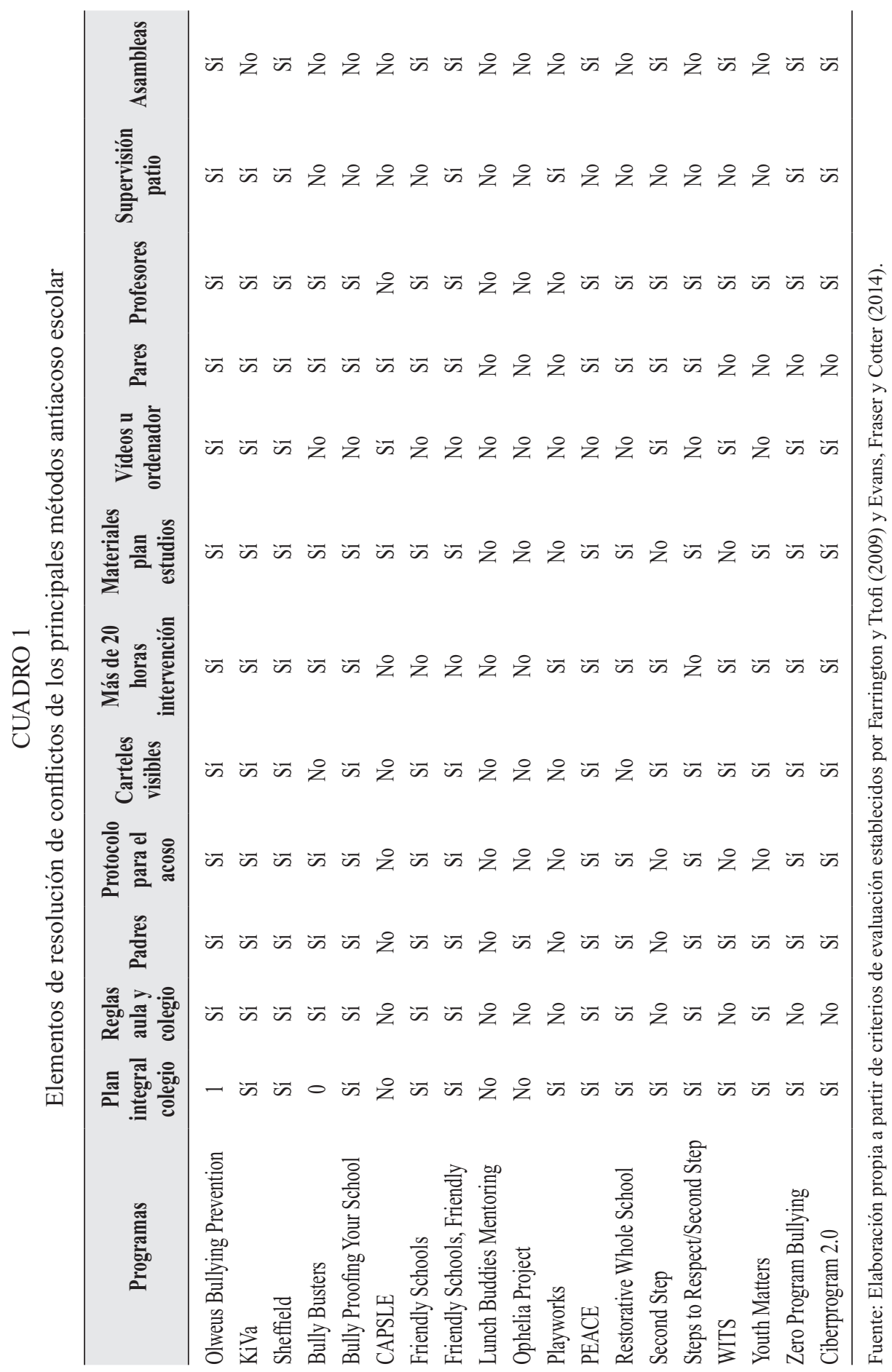


siones y reconocido a nivel internacional. El principal requisito metodológico para incluir los casos en el estudio consistió en el requerimiento de que incorporaran lo más nítidamente posible los efectos de los programas usados en la reducción del acoso escolar.

Tal como hacen otros autores, en este estudio se admitieron cuatro variaciones metodológicas:

1. Comparación de un grupo experimental pretest con otro postest, entendiendo por grupo experimental o semiexperimental el de aquellos jóvenes que se expusieron al programa independientemente de su asignación al azar.

2. Comparación de un grupo experimental que hubiera recibido la intervención con un grupo de control que no la hubiera recibido.

3. Estudios longitudinales con cohortes de edad diferentes.

4. Experimentos aleatorizados. Teniendo en cuenta que, como señalan Evans, Fraser y Cotter (2014), pocos estudios han medido el acoso escolar con la precisión suficiente como para que tenga validez de constructo, los efectos son relativamente poco significativos en experimentos aleatorios, aunque relativamente altos en los diseños cuasiexperimentales y de grupo de edad.

Los casos se seleccionaron de acuerdo a la objetividad, sistematización y replicabilidad de los resultados (Figura 1), así como a la descripción del programa utilizado. Entre las variables de control que se ha impuesto en la revisión sistemática de este estudio figuran la especificación de la reducción que obtuvo el programa utilizado, así como su constructo. Para escoger los estudios se ha recurrido a publicaciones recogidas en las principales bases de datos, así como a revistas especializadas y libros. Finalmente se redujeron a Cochrane (9832), PubMed (353) y Scopus (1591). Se incluyeron también los casos incluidos en 21 artículos referidos a metaanálisis y revisiones sistemáticas. En total, se obtuvieron 159 casos, de los cuales se seleccionaron 40 (Figura 1). La revisión sistemática llevada a cabo trató de minimizar errores en la confección de los programas utilizados.

\section{ANÁLISIS ESTADÍSTICO}

Los descriptivos de los tres programas indican una diferencia considerable entre los métodos integrales y los híbridos (Tabla 1). Para comprobar la eficacia de los tres programas en la reducción de acoso escolar se utilizará un test de varianza (o ANOVA) de un factor. 
FIGURA 1

Bibliografía sistemática internacional: acoso escolar (bullying), victimización (victimization)

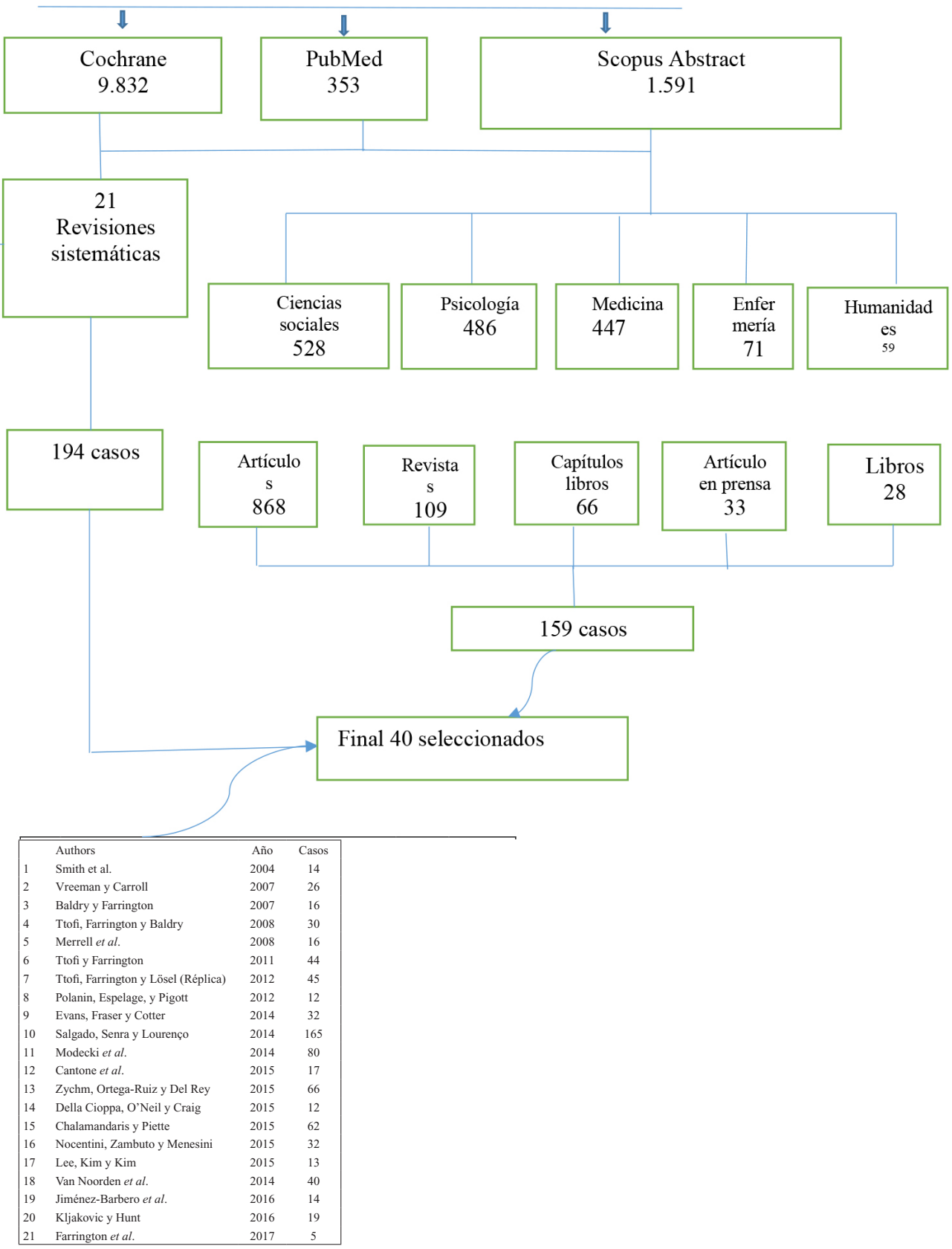


TABLA 1

Descriptivos de los tres programas por reducción de acoso escolar

\begin{tabular}{|c|c|c|c|c|c|c|c|c|}
\hline & \multirow[t]{2}{*}{ N. ${ }^{\circ}$} & \multirow[t]{2}{*}{ Media } & \multirow{2}{*}{$\begin{array}{c}\text { Desviación } \\
\text { estándar }\end{array}$} & \multirow{2}{*}{$\begin{array}{c}\text { Error } \\
\text { estándar }\end{array}$} & \multicolumn{2}{|c|}{$\begin{array}{l}95 \% \text { del intervalo } \\
\text { de confianza para } \\
\text { la media }\end{array}$} & \multirow[t]{2}{*}{ Mínimo } & \multirow[t]{2}{*}{ Máximo } \\
\hline & & & & & $\begin{array}{l}\text { Límite } \\
\text { inferior }\end{array}$ & $\begin{array}{c}\text { Límite } \\
\text { superior }\end{array}$ & & \\
\hline Reactivo & 19 & 23,85 & 11,92 & 2,73 & 18,10 & 29,60 & 3,00 & 48,00 \\
\hline Proactivo & 11 & 27,72 & 13,93 & 4,20 & 18,36 & 37,09 & 6,00 & 53,50 \\
\hline Híbrido & 10 & 12,15 & 5,65 & 1,78 & 8,10 & 16,19 & 5,00 & 23,50 \\
\hline Total & 40 & 21,99 & 12,59 & 1,99 & 17,96 & 26,02 & 3,00 & 53,50 \\
\hline
\end{tabular}

Las diferencias entre las medias se pueden apreciar con nitidez en la Figura 1.

\section{GRÁFICO 1}

Medias de los tres grupos

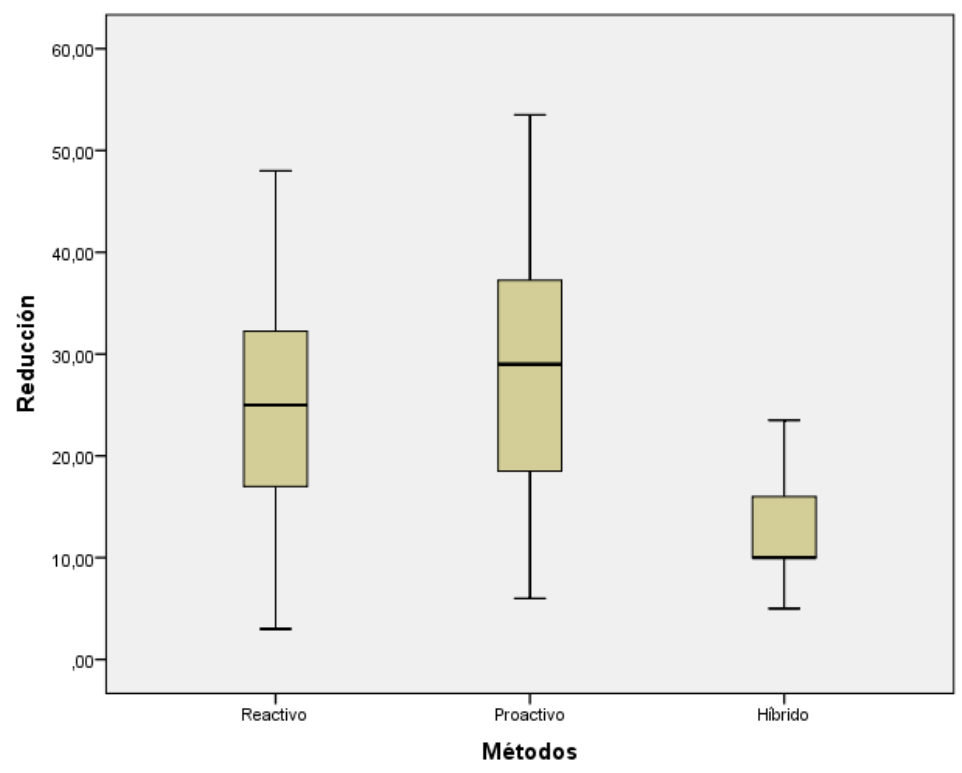

Las pruebas de normalidad se establecen mediante los test de Kolmogorov-Smirnov y Shapiro-Wilk (Tabla 2). 
TABLA 2

Pruebas de normalidad

\begin{tabular}{llccccccc}
\hline & \multirow{2}{*}{ Métodos } & \multicolumn{2}{c}{ Kolmogorov-Smirnov ${ }^{\text {a }}$} & \multicolumn{3}{c}{ Shapiro-Wilk } \\
\cline { 3 - 8 } & & Estadístico & gl & Sig. & Estadístico & gl & Sig. \\
\hline \multirow{2}{*}{ Reducción } & Reactivo & 0,22 & 19 & 0,014 & 0,92 & 19 & 0,11 \\
& Proactivo & 0,11 & 11 & $0,20^{*}$ & 0,98 & 11 & 0,97 \\
& Híbrido & 0,28 & 10 & 0,02 & 0,88 & 10 & 0,15 \\
\hline
\end{tabular}

* Esto es un límite inferior de la significación verdadera.

${ }^{a}$ Corrección de significación de Lilliefors

Al ser sigma mayor que 0,05, se rechaza la hipótesis de igualdad de varianzas, es decir, existe diferencia entre al menos dos de los grupos. Rechazamos la hipótesis nula y nos quedamos con la alternativa que sostiene que los grupos son distintos. Establecemos la homogeneidad de las medias mediante el test de Levene (Tabla 3).

TABLA 3

Prueba de Levene de homogeneidad de varianzas

\begin{tabular}{cccc}
\hline Estadístico de Levene & df1 & df2 & Sig. \\
\hline 2,73 & 2 & 37 & 0,07 \\
\hline
\end{tabular}

Existe un efecto estadísticamente significativo (Tabla 4) del hecho de reducción de acoso escolar por medio de los programas establecidos $\mathrm{F}(2,37)=5.391, \mathrm{p}<.05, \mathrm{n}^{2}=$ $1396.25 / 6187.88$

TABLA 4

Análisis de varianza de los tres programas

\begin{tabular}{lccccc}
\hline & Suma de cuadrados & gl & Media cuadrática & F & Sig. \\
\hline Entre grupos & 1396,25 & 2 & 698,12 & 5,39 & 0,009 \\
Dentro de grupos & 4791,63 & 37 & 129,50 & & \\
Total & 6187,88 & 39 & & & \\
\hline
\end{tabular}

Las pruebas de igualdad de medias de Welch y Brown-Forsythe ofrecen el siguiente resultado (Tabla 5). 
TABLA 5

Pruebas sólidas de igualdad de medias

\begin{tabular}{lllll}
\hline & \multicolumn{1}{c}{ Estadístico $^{\mathbf{a}}$} & df1 & df2 & Sig. \\
\hline Welch & 9.764 & 2 & 21,402 & 0,001 \\
Brown-Forsythe & 5.829 & 2 & 24,337 & 0,009 \\
\hline
\end{tabular}

${ }^{\text {a }} \mathrm{F}$ distribuida de forma asintótica.

Por lo tanto, se rechaza la hipótesis nula y se acepta la hipótesis alternativa que sostiene que existen diferencias estadísticamente significativas en las medias de los tres grupos. Para comprobar las diferencias acudimos a las pruebas de comparaciones múltiples (Tabla 6), las cuales indican que existen diferencias estadísticamente significativas entre los grupos reactivos e híbridos $\mathrm{t}(37)=2,63, \mathrm{p}<0,05$; entre los grupos pro

TABLA 6

Comparaciones múltiples de los tres programas

\begin{tabular}{|c|c|c|c|c|c|c|c|}
\hline & \multirow{2}{*}{$\begin{array}{l}\text { (I) } \\
\text { Métodos }\end{array}$} & \multirow{2}{*}{$\begin{array}{c}(\mathbf{J}) \\
\text { Métodos }\end{array}$} & \multirow{2}{*}{$\begin{array}{c}\text { Diferencia } \\
\text { de medias } \\
\text { (I-J) }\end{array}$} & \multirow{2}{*}{$\begin{array}{c}\text { Error } \\
\text { estándar }\end{array}$} & \multirow{2}{*}{ Sig. } & \multicolumn{2}{|c|}{$\begin{array}{c}95 \% \text { de intervalo } \\
\text { de confianza }\end{array}$} \\
\hline & & & & & & $\begin{array}{l}\text { Límite } \\
\text { inferior }\end{array}$ & $\begin{array}{l}\text { Límite } \\
\text { superior }\end{array}$ \\
\hline \multirow[t]{6}{*}{ HSD Tukey } & \multirow[t]{2}{*}{ Reactivo } & Proactivo & -3.87 & 4,31 & ,64 & $-14,40$ & 6,65 \\
\hline & & Híbrido & $11,70^{*}$ & 4,44 &, 03 &, 84 & 22,55 \\
\hline & \multirow{2}{*}{ Proactivo } & Reactivo & 3,87 & 4,31 & 64 & $-6,65$ & 14,40 \\
\hline & & Híbrido & $15,57^{*}$ & 4,97 &, 00 & 3,43 & 27,71 \\
\hline & \multirow[t]{2}{*}{ Híbrido } & Reactivo & $-11,70^{*}$ & 4,44 &, 03 & $-22,55$ &,- 84 \\
\hline & & Proactivo & $-15,57^{*}$ & 4,97 &, 00 & $-27,71$ & $-3,43$ \\
\hline \multirow[t]{6}{*}{ Bonferroni } & \multirow[t]{2}{*}{ Reactivo } & Proactivo & $-3,87$ & 4,31 & 1,00 & $-14,68$ & 6,93 \\
\hline & & Híbrido & $11,70^{*}$ & 4,44 &, 03 &, 55 & 22,85 \\
\hline & \multirow[t]{2}{*}{ Proactivo } & Reactivo & 3,87 & 4,31 & 1,00 & $-6,93$ & 14,68 \\
\hline & & Híbrido & $15,57^{*}$ & 4,97 &, 01 & 3,10 & 28,04 \\
\hline & \multirow[t]{2}{*}{ Híbrido } & Reactivo & $-11,70^{*}$ & 4,44 &, 03 & $-22,85$ &,- 55 \\
\hline & & Proactivo & $-15,57^{*}$ & 4,97 & 01 & $-28,04$ & $-3,10$ \\
\hline \multirow[t]{6}{*}{ Games-Howell } & \multirow[t]{2}{*}{ Reactivo } & Proactivo & $-3,87$ & 5,01 &, 72 & $-16,64$ & 8,89 \\
\hline & & Híbrido & $11,70 *$ & 3,26 &, 00 & 3,59 & 19,81 \\
\hline & \multirow[t]{2}{*}{ Proactivo } & Reactivo & 3,87 & 5,01 &, 72 & $-8,89$ & 16,64 \\
\hline & & Híbrido & $15,57^{*}$ & 4,56 &, 01 & 3,56 & 27,58 \\
\hline & \multirow[t]{2}{*}{ Híbrido } & Reactivo & $-11,70^{*}$ & 3,26 &, 00 & $-19,81$ & $-3,59$ \\
\hline & & Proactivo & $-15,57^{*}$ & 4,56 &, 01 & $-27,58$ & $-3,56$ \\
\hline
\end{tabular}

* La diferencia de medias es significativa en el nivel 0,05. 
activos e híbridos, $\mathrm{t}(37)=3,1, \mathrm{P}<0,05$; mientras que no existen diferencias entre los grupos reactivos y proactivos $\mathrm{t}(37)=3,1 \mathrm{p}>0,05$

La prueba HSD de Tukey (Tabla 7) muestra que hay diferencias entre el programa híbrido y los demás, ya que se encuentran en diferentes columnas y con valores de significación superiores a 0,05 .

TABLA 7

Prueba de Tukey para establecer subconjuntos homogéneos

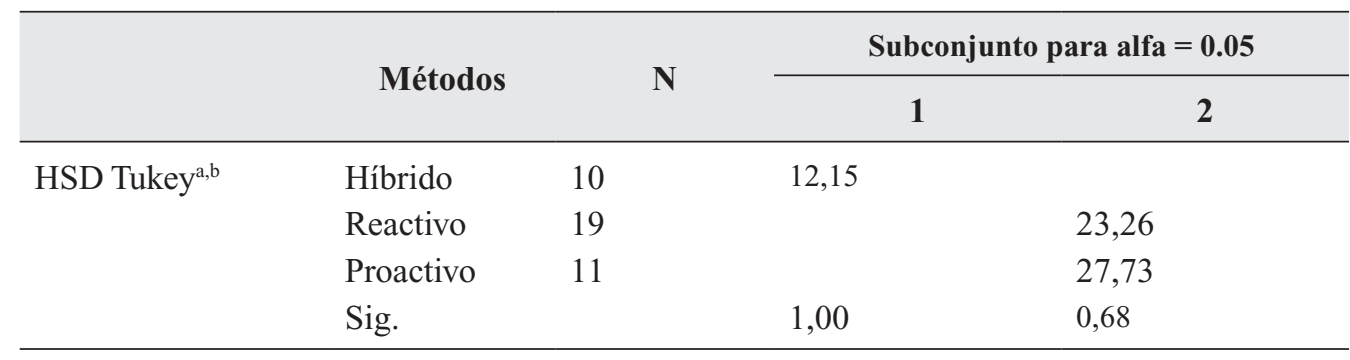

Se visualizan las medias para los grupos en los subconjuntos homogéneos.

a Utiliza el tamaño de la muestra de la media armónica $=12.318$.

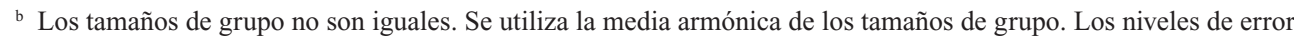
de tipo I no están garantizados.

\section{RESULTADOS:}

En total se han analizado 40 casos, que suponen 113.448 estudiantes, con una media de 2.836,2, una desviación típica de 4.497,75, un mínimo de 16 y un máximo de 23.430 estudiantes. En conjunto, entre los tres programas, supone una reducción del $21 \%$. El grupo que presenta una mayor reducción del acoso escolar es el proactivo, con una media del $27 \%$, seguido del reactivo, con una media del 23\%, y finalmente el híbrido, que alcanza el $12 \%$ de media. No existen muchas diferencias entre los tres grupos en cuanto a los valores mínimos: en los programas proactivos nunca bajan del $6 \%$, en los reactivos algún programa solo consiguió una reducción del 3\%, y en los híbridos, del 5\%.

La mayor diferencia se da, sin embargo, en el valor máximo, puesto que en el grupo proactivo existe un caso que alcanzó el 53\%, alejado del 48\% de un caso del reactivo y a mayor distancia del grupo híbrido, con un $23 \%$. Hay que tener en cuenta también que el grupo proactivo tiene una mayor desviación típica y error estándar que los otros dos grupos; es decir, el resultado es menos uniforme, lo cual indica que puede haber mucha distancia entre unos casos y otros. 
En la clasificación por países (Cuadro 2), durante los primeros años se aprecia un claro liderazgo de los nórdicos (Noruega y Finlandia), a los cuales se unieron posteriormente Reino Unido, Australia y Canadá, con programas innovadores en algunos puntos. En la década de los ochenta del siglo xx se produce la incorporación de Estados Unidos, propiciada por el cambio de paradigma en el tratamiento de la violencia escolar, posiblemente sobre la base del grave problema de los homicidios que se han producido en algunos de sus centros académicos. Los países representados en la muestra total son Alemania (1), Australia (2), Canadá (4), Estados Unidos (15), España (1), Finlandia (6), Irlanda (1), Italia (1), Nueva Zelanda (1), Noruega (5), Reino Unido (3) y Suiza (1).

\section{DISCUSIÓN}

Es posible que las cifras dependan de los programas seleccionados para formar parte de los grupos. Aun así, siempre se puede sostener que, tras la revisión de una serie de casos y su agrupación por criterios objetivos en tres grupos, el resultado obtenido constituye un primer avance en el conocimiento y la evaluación de los programas antiacoso escolar.

Una dificultad importante en esta tarea es la heterogeneidad observada entre los casos analizados en temas como la técnica utilizada, la definición de acoso escolar, el empleo del cuestionario y la publicación de resultados. El análisis de subgrupos demuestra que la heterogeneidad depende de comparar estudios de diferente complejidad, es decir, con tamaños de muestra más grandes, que evaluaron intervenciones multidisciplinares o globales, o que transcurren en un intervalo de tiempo más largo. En este sentido, algunos estudios han mencionado la dificultad de resolver esta variabilidad cuando existen programas que implican el análisis de muchas variables, porque deben considerarse como sistemas complejos (Burton, 2012). Por ello, la heterogeneidad en este tipo de estudios se debe estimar como la variación natural de los efectos observados. En el caso de la medida de la frecuencia del acoso escolar, la heterogeneidad entre los estudios también puede deberse a un sesgo de publicación, tal como se refleja en la asimetría observada en la exposición de los resultados y la metodología, en los componentes de la intervención, en el diseño de la evaluación, en el análisis estadístico y en la selección de información (Ttofi y Farrington, 2011).

Muchos estudios no publican el porcentaje de éxito o fracaso de sus programas y se limitan a reproducir el obtenido por Olweus en Noruega. Gran parte de los casos son bastante complejos, lo cual ha dificultado establecer clasificaciones, sobre todo a la hora de diferenciar entre los programas integrales reactivos y proactivos. Lo mismo cabe 
decir al establecer la cifra única de reducción de acoso escolar, puesto que muchos estudios muestran reducciones en varias modalidades de acoso escolar, lo que ha obligado a calcularla. Existen programas, como el expect to respect, con resultados muy contradictorios, ya que después de aplicarlos el porcentaje de acoso aumentó en el $60 \%$ en el grupo experimental y el 59\% en el grupo de control, aunque la victimización bajó el $10 \%$ en el grupo experimental y el $27 \%$ en el grupo de control.

El procedimiento más polémico es establecer una única cifra que englobe a todas las variaciones del acoso escolar, dado que es un fenómeno multifacético que incluye al menos ocho categorías, tanto para la agresión como para la victimización. El caso más paradigmático de un hecho similar se produce en el ámbito de la delincuencia; aunque en este caso los países suelen ofrecer datos globales, algunas Administraciones, como la francesa, se han negado a facilitarlos, por considerar que engloban a delitos tan distintos como los robos y los asesinatos.

En el acoso escolar ocurre algo parecido, puesto que de él existen muchas modalidades (pegar, insultar y reñir, romper, boicotear...). Pero además esta cifra es muy difícil de obtener, dada la tendencia a no proporcionarla de muchos estudios, concretamente en varios de los seleccionados. Junto a ello, hay que señalar que no son pocos los programas que incluyen varias cifras de medida temporal (4 meses, 1 año, 2 años), con lo que la validez de constructo vuelve a resultar inconsistente. Otra dificultad radica en la edad, que en muchos estudios no se publica con claridad. Se suele facilitar el grado de estudios, que al ser diferente en muchos países dificulta el establecimiento de esta variable. En este trabajo la cifra única de acoso escolar corresponde a diferentes poblaciones etarias.

CUADRO 2

Estudios representativos sobre aplicación de programas antiacoso escolar

\begin{tabular}{|c|c|c|c|c|c|c|c|c|c|}
\hline $\mathbf{N}$ & Estudio & Año & País & M & E. P. $\%$ & M & $\begin{array}{l}\text { Reducción } \\
\text { estimada* }\end{array}$ & Programa & Autores \\
\hline 1 & SAVE & 1995 & España & 910 & 50 & $\mathrm{H}$ & 10,00 & Híbrido & $\begin{array}{l}\text { Ortega, Del Rey y } \\
\text { Mora-Merchan (2004) }\end{array}$ \\
\hline 2 & WITS & 2003 & Canada & 432 & 50 & $\mathrm{H}$ & 10,00 & Híbrido & $\begin{array}{l}\text { Hoglund, Hosan y } \\
\text { Leadbeater (2012) }\end{array}$ \\
\hline 3 & Toronto & 2004 & Canadá & 898 & 100 & $\mathrm{H}$ & 5,00 & Híbrido & $\begin{array}{l}\text { Pepler, Smith y Rigby } \\
\text { (2004) }\end{array}$ \\
\hline 4 & Youth matters & 2007 & EE. UU. & 1.126 & 75 & $\mathrm{H}$ & 16,00 & Híbrido & $\begin{array}{l}\text { Jenson y Dieterich } \\
(2007)\end{array}$ \\
\hline 5 & CAPSLE & 2009 & EE. UU. & 1.345 & 75 & $\mathrm{H}$ & 19,00 & Híbrido & Fonagy et al (2009) \\
\hline 6 & FearNot! & 2009 & R.U./Alemania & 1.129 & 25 & $\mathrm{H}$ & 10,00 & Híbrido & Sapouna et al. 2010 \\
\hline 7 & Lunch Buddies & 2011 & EE. UU. & 36 & - & $\mathrm{H}$ & 11,00 & Híbrido & Elledge et al. (2011) \\
\hline 8 & Restorative & 2011 & China & 1.176 & 50 & $\mathrm{H}$ & 50,00 & Híbrido & Wong et al. (2011) \\
\hline
\end{tabular}




\begin{tabular}{|c|c|c|c|c|c|c|c|c|c|}
\hline N & Estudio & Año & País & M & E. P. \% & M & $\begin{array}{l}\text { Reducción } \\
\text { estimada* }\end{array}$ & Programa & Autores \\
\hline 9 & Playworks & 2012 & EE. UU & 1.982 & 25 & $\mathrm{H}$ & 10,00 & Híbrido & Bleeker et al. (2012) \\
\hline 10 & Ophelia & 2012 & EE. UU. & 18 & -- & $\mathrm{H}$ & 7,00 & Híbrido & Wright y Li (2012) \\
\hline 11 & Second Step & 2013 & EE. UU. & 3.616 & 75 & $\mathrm{H}$ & 42,00 & Híbrido & Espelage et al. (2013) \\
\hline 12 & Ciberprogra 2.0 & 2014 & España & 176 & -- & $\mathrm{H}$ & 35,00 & Híbrido & $\begin{array}{l}\text { Garaigordobil y } \\
\text { Martinez (2016) }\end{array}$ \\
\hline 13 & Friendly Scho & 2000 & Australia & 2.068 & 75 & $\mathrm{H}$ & 23,5 & Híbrido & Cross et al. (2004). \\
\hline 14 & Toronto & 1990 & Canadá & 758 & 50 & $\mathrm{P}$ & 34,00 & Proactivo & Pepler et al. (2004) \\
\hline 15 & Finnish (1) & 1999 & Finlandia & 16 & 75 & $\mathrm{P}$ & 22,00 & Proactivo & $\begin{array}{l}\text { Salmivalli, Kaukiainen } \\
\text { y Voeten }(2005)\end{array}$ \\
\hline 16 & Finnish (2) & 2001 & Finlandia & 1.220 & 100 & $\mathrm{P}$ & 34,00 & Proactivo & Salmivalli et al. (2004) \\
\hline 17 & Chula Vista & 2007 & EE. UU. & 3.378 & 100 & $\mathrm{P}$ & 17,00 & Proactivo & $\begin{array}{l}\text { Pagliocca, Limber y } \\
\text { Hashima (2007) }\end{array}$ \\
\hline 18 & PEACE & 2007 & Australia & 954 & 100 & $\mathrm{P}$ & 13,00 & Proactivo & $\begin{array}{l}\text { Slee y Mohyla (2007) } \\
\text { japón }\end{array}$ \\
\hline 19 & Steps Respect & 2011 & EE. UU. & 2.940 & 75 & $\mathrm{P}$ & 40,50 & Proactivo & Brown et al. (2011) \\
\hline 20 & KiVa & 2011 & Finlandia & 8.237 & 100 & $\mathrm{P}$ & 53,50 & Proactivo & Kärnä et al. (2011). \\
\hline 21 & Kiva (nacio) & 2011 & Finlandia & 150.000 & 100 & $\mathrm{P}$ & 15,00 & Proactivo & Karnä et al. (2011) \\
\hline 22 & Positive & 2011 & Chicago & 510 & 100 & $\mathrm{P}$ & 41,00 & Proactivo & $\begin{array}{l}\text { Li, Cross y Smith } \\
\text { (2011) }\end{array}$ \\
\hline 23 & KiVa & 2011 & Finlandia & 5.651 & 100 & $\mathrm{P}$ & 29,00 & Proactivo & $\begin{array}{l}\text { Salmivalli, Kärnä y } \\
\text { Poskiparta (2011) }\end{array}$ \\
\hline 24 & KiVa & 2013 & Finlandia & 23.430 & 100 & $\mathrm{P}$ & 20,00 & Proactivo & Kärnä et al. (2013) \\
\hline 25 & Kiva (Gales) & 2016 & R Unido & 2.140 & 100 & $\mathrm{P}$ & 6,00 & Proactivo & Clarkson et al. (2015). \\
\hline 26 & Bergen 1. & 1983 & Noruega & 2.500 & 50 & $\mathrm{D}$ & 28,00 & Reactivo & Olweus y Limber (2010) \\
\hline 27 & Sheffield & 1991 & ReinoUnido & 6.500 & 100 & $\mathrm{D}$ & 25,00 & Reactivo & Smith y Sharp (1994). \\
\hline 28 & Olweus Alema & 1994 & Alemania & 14.788 & 75 & $\mathrm{D}$ & 10,00 & Reactivo & Hanewinkel, (2004) \\
\hline 29 & Carolina & 1994 & EE. UU. & 6.389 & 75 & $\mathrm{D}$ & 25,00 & Reactivo & Melton et al. (1998). \\
\hline 30 & Expect Respe & 1994 & EE. UU. & 929 & -- & $\mathrm{D}$ & 3,00 & Reactivo & Rosenbluth et al. (2004) \\
\hline 31 & The Flemish & 1994 & Finlandia & 1.104 & 75 & $\mathrm{D}$ & 6,00 & Reactivo & $\begin{array}{l}\text { Stevens, Bourdeaudhuij } \\
\text { y Oost (2000) }\end{array}$ \\
\hline 32 & Be-Prox & 1997 & Suiza & 152 & 75 & $\mathrm{D}$ & 15,00 & Reactivo & $\begin{array}{l}\text { Alsaker y Valkanover } \\
\text { (2012) }\end{array}$ \\
\hline 33 & Bergen 2 & 1997 & Noruega & 3.200 & 100 & $\mathrm{D}$ & 23,00 & Reactivo & $\begin{array}{l}\text { Olweus y Limbert } \\
\text { (2010) Olweus (2004) }\end{array}$ \\
\hline 34 & Donegal & 1998 & Irlanda & 527 & 100 & $\mathrm{D}$ & 32,50 & Reactivo & $\begin{array}{l}\text { O'Moore y Minton } \\
\text { (2004) }\end{array}$ \\
\hline 35 & Steps Respect & 2001 & EE. UU. & 1.126 & 75 & $\mathrm{D}$ & 25,00 & Reactivo & $\begin{array}{l}\text { Frey, Edstrom y } \\
\text { Hirschstein (2005). }\end{array}$ \\
\hline 36 & Oslo 1-2 & 2001 & Noruega & 900 & 100 & $\mathrm{D}$ & 48,00 & Reactivo & Olweus (2004) \\
\hline 37 & Bulli Pupe & 2004 & Italia & 239 & 75 & $\mathrm{D}$ & 33,00 & Reactivo & $\begin{array}{l}\text { Baldry y Farrington } \\
(2007)\end{array}$ \\
\hline 38 & Dare to Care & 2004 & Canadá & 197 & 75 & $\mathrm{D}$ & 19,00 & Reactivo & $\begin{array}{l}\text { Beran, Tutty y Steinrath } \\
\text { (2004) }\end{array}$ \\
\hline 39 & Olweus National & 2006 & Noruega & 8.388 & 100 & $\mathrm{D}$ & 33,00 & Reactivo & Olweus y Limber (2010) \\
\hline 40 & Seattle & 2007 & EE. UU. & 4.959 & 100 & $\mathrm{D}$ & 32,00 & Reactivo & $\begin{array}{l}\text { Bauer, Lozano y Rivara } \\
\text { (2007). }\end{array}$ \\
\hline 41 & Philadelphia & 2007 & EE. UU. & 456 & 100 & $\mathrm{D}$ & 3,00 & Reactivo & Black y Jackson (2007) \\
\hline
\end{tabular}




\begin{tabular}{llrlllllll}
\hline N & \multicolumn{1}{c}{ Estudio } & Año & País & M & E. P. \% & M $\begin{array}{l}\text { Reducción } \\
\text { estimada* }\end{array}$ & Programa & \multicolumn{1}{c}{ Autores } \\
\hline $\mathbf{4 2}$ & Build Respect & 2007 & EE. UU. & 1.237 & 100 & D & 33,70 & Reactivo & Evers et al. (2007) \\
$\mathbf{4 3}$ & Zero & 2010 & Noruega & 409 & 100 & D & 25,00 & Reactivo & Roland et al. (2010) \\
44 & Olweus USA & 2011 & EE. UU. & 270 & 100 & D & 32,50 & Reactivo & Bowllan (2011) \\
\hline
\end{tabular}

Fuente: Elaboración propia a partir del banco de datos. Nota. $\mathrm{N}=$ número de estudio, $\mathrm{M}=$ muestra. E.P. $=$ elementos programa $=$ formación de padres, formación de profesores, formación de personal auxiliar, supervisión de patio escolar y lugares remotos, vídeos, libros, programas de ordenador. $\mathrm{D}=$ diseño de estudio: $\mathrm{E}=$ experimental pretes/postest, $\mathrm{S}=$ aleatorio, $\mathrm{C}=$ cohorte de edad, $\mathrm{O}=$ otros). $\mathrm{M}=\mathrm{D}$ (reactivo), $\mathrm{P}$ (proactivo), $\mathrm{S}$ (híbrido). $\mathrm{R}=$ reducción estimada.

\section{CONCLUSIONES}

Con la irrupción del reconocimiento del acoso escolar, los centros educativos han conocido una gran transformación que ha puesto en tela de juicio sus estructuras. El currículum ha dejado de ser oculto. Después de analizar cientos de programas y seleccionar los más representativos, se puede afirmar que existe una gran evidencia empírica de la eficacia de los métodos antiacoso escolar desde sus inicios en 1983 con la propuesta innovadora y revolucionaria de Olweus. Numerosas publicaciones de diferente tipo han documentado su valía. Se han aplicado en diferentes culturas, en diferentes escenarios, en diferentes estamentos políticos y con modificaciones adaptativas a los centros o a los requerimientos de diferentes países. Aunque se podría decir que existen tanto prototipos como centros escolares que los aplican por su gran versatilidad, las diferentes aplicaciones reconocidas académicamente han seguido tres grandes modelos de intervención: integrales reactivos, integrales proactivos y parciales o híbridos. No obstante, a pesar del marcado incremento en la investigación de antiacoso escolar, todavía se necesita aprender sobre cómo diseñar e implementar programas de intervención efectiva, sobre todo teniendo en cuenta los diferentes resultados de investigación de intervención a través de estudios en diferentes países. ¿De qué manera y por qué un programa antiacoso es más efectivo que otro? ¿Qué elementos de la intervención pueden predecir el éxito de un programa en la reducción de la intimidación escolar? Y que programa es el más efectivo para cada centro escolar. Estas básicas preguntas han inspirado nuestra investigación.

Los procedimientos integrales reactivos basados en el método OBPP han demostrado su eficacia en la mayor parte de las experiencias publicadas con excepción de Noruega (Roland) y Alemania. Se podría decir que los métodos alternativos o métodos integrales proactivos como el Kiva, incorporados en la última década con un gran éxito, 
mantienen los mismos estándares que los anteriores y solo contemplan una parte de variación, toda vez que muchos elementos son reconocidos como comunes, como la necesidad de aplicar un método integral basado en cuatro ámbitos de actuación, la detección del problema por medio de un cuestionario o el reconocimiento de la necesidad de mejorar los espacios donde se produce el acoso. Dado que estos dos grandes modelos incorporan una prevención, una intervención y una resolución de los conflictos, la diferencia entre ellos se produce en aspectos filosóficos o de diseño de la intervención. Junto a estos dos grandes modelos se han desarrollado a lo largo del tiempo otras intervenciones parciales consistentes solo en los problemas relacionales basados en conceptos de convivencia alternativos al uso de sanciones, por lo que en muchas ocasiones han sido incorporados a los dos modelos anteriores como una parte de su actuación.

\section{BIBLIOGRAFÍA}

Alsaker, F. D. y Valkanover, S. (2001). "Early diagnosis and prevention of victimization in kindergarten", en Juvonen, J., \& Graham, S. (coords.), Peer harassment in school: The plight of the vulnerable and victimized, Nueva York: Guilford Press.

Baldry, A. C. y Farrington, D. P. (2007). "Evaluation of an intervention program for the reduction of bullying and victimization in schools", Aggressive Behavior, 30(1): 1-15. https://doi.org/10.1002/ab.20000

Bauer, N. S., Lozano, P. y Rivara, F. P. (2007). "The effectiveness of the Olweus Bullying Prevention Program in public middle schools: A controlled trial", Journal of Adolescent Health 40(3): 266-274. https://doi.org/10.1016/j.jadohealth.2006.10.005

Beran, T. N., Tutty, L. y Steinrath, G. (2004). "An evaluation of a bullying prevention program for elementary schools", Canadian Journal of School Psychology 19(1-2): 99-116. https://doi.org/10.1177/082957350401900105

Black, S. A. y Jackson, E. (2007). "Using bullying incident density to evaluate the Olweus Bullying Prevention Programme”, School Psychology International 28(5): 623-638. https://doi.org/10.1177/0143034307085662

Bleeker, M., James-Burdumy, S., Beyler, N., Dodd, A. H., London, R. A., Westrich, L., ... y Castrechini, S. (2012). Findings from a Randomized Experiment of Playworks: Selected Results from Cohort 1. Princenton, NJ: Mathematica Policy Research.

Bowllan, N. M. (2011). Implementation and evaluation of a comprehensive, school-wide bullying prevention program in an urban/suburban middle school", Journal of School Health 81(4): 167-173. https://doi.org/10.1111/j.1746-1561.2010.00576.x 
Brown, E. C., Low, S., Smith, B. H. y Haggerty, K. P. (2011). "Outcomes from a school-randomized controlled trial of steps to respect: A bullying prevention program", School Psychology Review 40 (3) : 423-443.

Cantone, E., Piras, A. P., Vellante, M., Preti, A., Daníelsdóttir, S., D’Aloja, E., ... y Bhugra, D. (2015). "Interventions on bullying and cyberbullying in schools: A systematic review", Clinical practice and epidemiology in mental health: $C P \& E M H, 11$ (Suppl 1 M4), 58.

Chalamandaris, A. G. y Piette, D. (2015). "School-based anti-bullying interventions: Systematic review of the methodology to assess their effectiveness". Aggression and violent behavior 24: 131-174. https://doi.org/10.1016/j.avb.2015.04.004

Clarkson, S., Axford, N., Berry, V., Edwards, R. T., Bjornstad, G., Wrigley, Z., ... y Hutchings, J. 2015. "Effectiveness and micro-costing of the KiVa school-based bullying prevention programme in Wales: study protocol for a pragmatic definitive parallel group cluster randomised controlled trial". BMC public health 16(1): 104. https:// doi.org/10.1186/s12889-016-2746-1

Cowie, H., y Hutson, N. (2005). "Peer support: A strategy to help bystanders challenge school bullying", Pastoral Care in Education 23(2): 40-44.

Cross, D., Hall, M., Hamilton, G., Pintabona, Y. y Erceg, E. (2004). “Australia: The Friendly Schools Project"v, en Smith, Peter K., Debra Pepler, and Ken Rigby (coords.): Bullying in schools: How successful can interventions be?. New York: Cambridge University Press.

Della Cioppa, V., O’Neil, A. y Craig, W. (2015). "Learning from traditional bullying interventions: A review of research on cyberbullying and best practice", Aggression and Violent Behavior (23): 61-68. https://doi.org/10.1016/j.avb.2015.05.009

Elledge, C. L., Cavell, T. A., Ogle, N. T. y Newgent, R. A. (2010). "School-based mentoring as selective prevention for bullied children: A preliminary test", The journal of primary prevention 31(3) : 171-187. https://doi.org/10.1007/s10935-010-0215-7

Evans, C. B., Fraser, M. W. y Cotter, K. L. (2014). "The effectiveness of school-based bullying prevention programs: A systematic review”, Aggression and Violent Behavior 19(5): 532-544. https://doi.org/10.1016/j.avb.2014.07.004

Evers, K. E., Prochaska, J. O., Van Marter, D. F., Johnson, J. L. y Prochaska, J. M. (2007). "Transtheoretical-based bullying prevention effectiveness trials in middle schools and high schools", Educational Research 49(4): 397-414. https://doi. org/10.1080/00131880701717271

Farrington, D. P., Gaffney, H., Lösel, F. y Ttofi, M. M. (2017). "Systematic reviews of the effectiveness of developmental prevention programs in reducing delinquency, 
aggression, and bullying". Aggression and Violent Behavior 3(3): 91-106. https:// doi.org/10.1016/j.avb.2016.11.003

Farrington, D. P., y Ttofi, M. M. (2009). "School-based programs to reduce bullying and victimization", The Campbell Collaboration (6):1-149. https://doi.org/10.4073/ csr.2009.6

Fonagy, P., Twemlow, S. W., Vernberg, E. M., Nelson, J. M., Dill, E. J., Little, T. D. y Sargent, J. A. (2009). "A cluster randomized controlled trial of child-focused psychiatric consultation and a school systems-focused intervention to reduce aggression", Journal of Child Psychology and Psychiatry 50(5): 607-616. https://doi.or$\mathrm{g} / 10.1111 / \mathrm{j} .1469-7610.2008 .02025 . \mathrm{x}$

Frey, K. S., Edstrom, L.V.S. y Hirschstein, M. K. (2005). “The Steps to Respect program uses a multi-level approach to reduce playground bullying and destructive playground behaviours", en Proceedings of Persistently Safe Schools. Washington, DC: Hamilton Fish Institute, George Washington University.

Gregory, A., Cornell, D., Fan, X., Sheras, P., Shih, T. H., y Huang, F. (2010). "Authoritative school discipline: High school practices associated with lower bullying and victimization", Journal of Educational Psychology 102(2): 483-496. https://doi. org/10.1037/a0018562

Hanewinkel, R. (2004). "Prevention of bullying in German schools: An evaluation of an anti-bullying approach", en Smith, Peter K., Debra Pepler, and Ken Rigby (coords). Bullying in schools: How successful can interventions be? Nueva York: Cambridge University Press.

Hoglund, W. L., Hosan, N. E. y Leadbeater, B. J. (2012). "Using Your WITS: A 6-Year Follow-Up of a Peer Victimization Prevention Program”, School Psychology Review 41(2): 193-214.

Hopkins, B., Maines, B. y Robinson, G. (2011). The restorative classroom: Using restorative approaches to foster effective learning. London: Optimus Education.

Jenson, J. M. y Dieterich, W. A. (2007). "Effects of a skills-based prevention program on bullying and bully victimization among elementary school children", Prevention Science 8(4): 285-296. https://doi.org/10.1007/s11121-007-0076-3

Jiménez-Barbero, J. A., Ruiz-Hernández, J. A., Llor-Zaragoza, L., Pérez-García, M. y Llor-Esteban, B. (2016). "Effectiveness of anti-bullying school programs: A meta-analysis", Children and Youth Services Review (61): 165-175. https://doi.org/10.1016/j.childyouth.2015.12.015

Kärna, A., Voeten, M., Little, T. D., Poskiparta, E., Kaljonen, A. y Salmivalli, C. (2011). „A large-scale evaluation of the KiVa antibullying program: Grades 4-6”, Child development 82(1): 311-330. https://doi.org/10.1111/j.1467-8624.2010.01557.x 
Kärna, A., Voeten, M., Little, T. D., Alanen, E., Poskiparta, E. y Salmivalli, C. (2013). "Effectiveness of the KiVa Antibullying Program: Grades 1-3 and 7-9". Journal of Educational Psychology 105(2): 535-551. https://doi.org/10.1037/a0030417

Kljakovic, M. y Hunt, C. (2016). "A meta-analysis of predictors of bullying and victimisation in adolescence. "Journal of adolescence 49: 134-145. https://doi.or$\mathrm{g} / 10.1016 / \mathrm{j}$.adolescence.2016.03.002

Lee, S., Kim, C. J. y Kim, D. H. (2015). "A meta-analysis of the effect of school-based anti-bullying programs", Journal of child health care 19(2): 136-153. https://doi. org/10.1177/1367493513503581

Li, Q., Cross, D. y Smith, P. K. (eds.) (2011). Cyberbullying in the global playground: Research from international perspectives. West Sussex: Wiley-Blackwell.

Melton, G. B., Limber, S. P., Cunningham, P., Osgood, W., Chambers, J., Flerx, V., ... y Nation, M. (1998). "Violence among rural youth: Final report to the Office of Juvenile Justice and Delinquency Prevention", Washington, DC: US Department of Justice, Office of Justice Programs, Office of Juvenile Justice and Delinquency Prevention.

Merrell, K. W., Gueldner, B. A., Ross, S. W. y Isava, D. M. (2008). "How effective are school bullying intervention programs? A meta-analysis of intervention research", School Psychology Quarterly, 23: 26-42. https://doi.org/10.1037/1045-3830.23.1.26

Modecki, K. L., Minchin, J., Harbaugh, A. G., Guerra, N. G. y Runions, K. C. (2014). "Bullying prevalence across contexts: A meta-analysis measuring cyber and traditional bullying", Journal of Adolescent Health 55(5): 602-611. https:/doi.org/10.1016/j.jadohealth.2014.06.007

Nocentini, A., Zambuto, V. y Menesini, E. (2015). "Anti-bullying programs and Information and Communication Technologies (ICTs): A systematic review", Aggression and Violent Behavior 23:52-60. https://doi.org/10.1016/j.avb.2015.05.012

Olweus, D. (2004). "The Olweus Bullying Prevention Programme: Design and implementation issues and a new national initiative in Norway", en Smith, Peter K., Debra Pepler, and Ken Rigby, (coords.) Bullying in schools: How successful can interventions be? Nueva York: Cambridge University Press.

Olweus, D. y Limber, S. P. (2010). "Bullying in school: Evaluation and dissemination of the Olweus Bullying Prevention Program", American Journal of Orthopsychiatry 80(1): 124-134. https://doi.org/10.1111/j.1939-0025.2010.01015.x

Ortega, R., Del Rey, R. y Mora-Merchan, J. A. (2004). "9 SAVE model: an anti-bullying intervention in Spain", en Smith, P.K., Pepler, D., and Rigby, K. (coords.), Bullying in schools: How successful can interventions be? NuevaYork: Cambridge University Press. 
O’Moore, A. M. y Minton, S. J. (2004). “Ireland: The Donegal Primary Schools' anti-bullying project", en Smith, P.K., Pepler, D. and Rigby, K. (coords.), Bullying in schools: How successful can interventions be? Nueva York: Cambridge University Press.

Pagliocca, P. M., Limber, S. P. y Hashima, P. (2007).Evaluation report for the Chula Vista Olweus Bullying Prevention Program. Final report prepared for the Chula Vista Police Department.

Pepler, D., Smith, P. K. y Rigby, K. (2004). "16 Looking back and looking forward: implications for making interventions work effectively", en Smith, P.K., Pepler, D., and Rigby, K. (coords.), Bullying in schools: How successful can interventions be? Nueva York: Cambridge University Press.

Pepler, D., Wendy, M., Craig, P. O. C., Atlas, R., y Charach, A. (2004). "Making a difference in bullying: Evaluation of a systemic school-based programme", en Smith, P.K., Pepler, D. and Rigby, K. (coords.), Bullying in schools: How successful can interventions be? Nueva York: Cambridge University Press.

Pikas, A. (1989). "A pure concept of mobbing gives the best results for treatment", School Psychology International 10(2): 95-104. https://doi. org/10.1177/0143034389102003

Polanin, J. R., Espelage, D. L. y Pigott, T. D. (2012). "A meta-analysis of school-based bullying prevention programs' effects on bystander intervention behavior", School Psychology Review 41(1): 47.

Rigby, K. (2013). "Bullying in schools and its relation to parenting and family life", Family Matters 92:61-67. Consulta, 12 de diciembre de 2017 (http://search.informit.com.au/documentSummary;dn=442237495856995;res=IELHSS)

Roland, E., Bru, E., Midthassel, U. V. y Vaaland, G. S. (2010). “The Zero programme against bullying: Effects of the programme in the context of the Norwegian manifesto against bullying", Social Psychology of Education 13(1): 41-55. https://doi. org/10.1007/s11218-009-9096-0

Rosenbluth, B., Whitaker, D. J., Sánchez, E. y Valle, L. A. (2004). “The Expect Respect project: Preventing bullying and sexual harassment in US elementary schools", en Smith, P.K., Pepler, D. and Rigby, K. (coords.), Bullying in schools: How successful can interventions be? Nueva York: Cambridge University Press.

Salgado, F. S., Senra, L. X. y Lourenço, L. M. (2014). "Effectiveness indicators of bullying intervention programs: A systematic review of the international literature", Estudos de Psicologia (Campinas) 31(2): 179-190. https://doi.org/10.1590/0103-16 6X2014000200004 
Salmivalli, C., Kaukiainen, A., Voeten, M. y Sinisammal, M. (2004). "Targeting the group as a whole: The Finnish anti-bullying intervention", en Smith, P.K., Pepler, D. and Rigby, K. (coords.), Bullying in schools: How successful can interventions be? Nueva York: Cambridge University Press.

Salmivalli, C., Kaukiainen, A. y Voeten, M. (2005). "Anti-bullying intervention: Implementation and outcome", British Journal of Educational Psychology 75(3): $465-$ 487. https://doi.org/10.1348/000709905X26011

Salmivalli, C., Kärnä, A. y Poskiparta, E. (2011). "Counteracting bullying in Finland: The KiVa program and its effects on different forms of being bullied", International Journal of Behavioral Development 35(5): 405-411. https://doi. org/10.1177/0165025411407457

Sapouna, M., Wolke, D., Vannini, N., Watson, S., Woods, S., Schneider, W., ... y Dautenhahn, K. (2010). "Virtual learning intervention to reduce bullying victimization in primary school: a controlled trial", Journal of Child Psychology and Psychiatry 51(1): 104-112. https://doi.org/10.1111/j.1469-7610.2009.02137.x

Slee, P. T. y Mohyla, J. (2007). "The PEACE Pack: an evaluation of interventions to reduce bullying in four Australian primary schools", Educational Research 49(2): 103-114. https://doi.org/10.1080/00131880701369610

Smith P. K. y Sharp S. (1994). "The problem of school bullying”, en Sharp, S. and Smith, P. School bullying: Insights and perspectives. London: Routledge. https://doi. org/10.4324/9780203425497

Smith, P. K., Salmivalli, C. y Cowie, H. (2012). "Effectiveness of school-based programs to reduce bullying: a commentary", Journal of Experimental Criminology 8(4): 433-441. https://doi.org/10.1007/s11292-012-9142-3

Smith, D. J., Schneider, B. H., Smith, P. K. y Ananiadou, K. (2004). "The effectiveness of whole school antibullying programs: A synthesis of evaluation research", School psychology review 33(4): 547-560.

Stevens, V., Bourdeaudhuij, I. y Oost, P. (2000)",Bullying in Flemish schools: An evaluation of anti-bullying intervention in primary and secondary schools", British Journal of Educational Psychology 70(2): 195-210. https://doi. org/10.1348/000709900158056

Thompson, F. y Smith, P. K. (2011). "The use and effectiveness of anti-bullying strategies in schools", Research Brief DFE-RR098. 1-220.

Ttofi, M. M., Farrington, D. P. y Lösel, F.(2012). "School bullying as a predictor of violence later in life: A systematic review and meta-analysis of prospective longitudinal studies", Aggression and Violent Behavior 17(5): 405-418. https://doi.org/10.1016/j. avb.2012.05.002. 
Ttofi, M. M. y Farrington, D P. (2011). "Effectiveness of school-based programs to reduce bullying: A systematic and meta-analytic review", Journal of Experimental Criminology 7(1): 27-56. https://doi.org/10.1007/s11292-010-9109-1.

Ttofi, M. M.; Farrington, D. P. y Baldry, A. C. (2008). Effectiveness of programmes to reduce school bullying: A systematic review: Report prepared for the Swedish National Council for Crime Prevention. Swedish National Council for Crime Prevention.

United Nations (1991) United Nations Convention on the Rights of the Child. Innocenti Studies. Florencia: UNICEF.

Valdés-Cuervo, Á. A., Martínez-Ferrer, B. y Carlos-Martínez, E. A. (2017). "El rol de las prácticas docentes en la prevención de la violencia escolar entre pares", Revista de Psicodidáctica 23(1): 33-38. https://doi.org/10.1387/RevPsicodidact.16434

Van Noorden, T. H., Haselager, G. J., Cillessen, A. H. y Bukowski, W. M. (2014). “Dehumanization in children: The link with moral disengagement in bullying and victimization", Aggressive behavior 40(4): 320-328. https://doi.org/10.1002/ab.21522

Vreeman, R. C. y Carroll, A. E. (2007). "A systematic review of school-based interventions to prevent bullying", Archives of Pediatrics \& Adolescent Medicine 161(1): 78-88. https://doi.org/10.1001/archpedi.161.1.78

Wright, M. F. y Li, Y. (2012). "Kicking the digital dog: A longitudinal investigation of young adults' victimization and cyber-displaced aggression", Cyberpsychology, Behavior, and Social Networking 15(9): 448-454. https://doi.org/10.1089/cyber.2012.0061

Yeager, D. S., Fong, C. J., Lee, H. Y. y Espelage, D. L. (2015). "Declines in efficacy of anti-bullying programs among older adolescents: Theory and a three-level meta-analysis", Journal of Applied Developmental Psychology 37: 36-51. https://doi. org/10.1016/j.appdev.2014.11.005

Zych, I., Ortega-Ruiz, R. y Del Rey, R. (2015). "Scientific research on bullying and cyberbullying: Where have we been and where are we going", Aggression and violent behavior (24): 188-198. https://doi.org/10.1016/j.avb.2015.05.015 